\title{
Modeling the Method of Laser Doppler Speckle-Velocimetry for Flat Objects with Retroreflective Surface
}

\author{
Gregory Dolya ${ }^{1} \Varangle$ \\ Olena Lytvynova ${ }^{2}$ \\ Faculty of Computer Science, V.N. Karazin Kharkiv National University, Kharkiv, Ukraine \\ E-mail:sdolva@ukr.net Tel: +38(057)7075022 \\ E-mail: litvinova.virt@gmail.com Tel: +38(067)57751.80
}

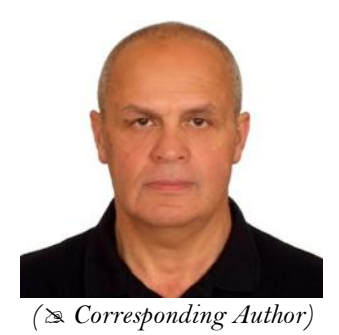

\begin{abstract}
The method of laser speckle-velocimetry based on the Doppler effect is considered. To increase the received signal power from a moving object, it is suggested to use a retro reflective sheeting in the form of a set of microscopic glass beads. A mathematical model of the proposed method is described. Physical simulation was carried out. The dependence of the power of the received signal on the frequency was obtained. The cases, when the values of the angles of incidence laser beam and the velocity of a moving object vary, were considered.
\end{abstract}

Keywords: Laser speckle-velocimetry, Doppler frequency, Retro reflective sheeting, Microscopic glass beads.

Citation | Gregory Dolya; Olena Lytvynova (2017). Modeling the Method of Laser Doppler Speckle-Velocimetry for Flat Objects with Retroreflective Surface. Asian Engineering Review, 4(2): 7-13. History:

Received: 12 October 2017

Revised: 1 November 2017

Accepted: 20 December 2017

Accepted: 20 December 2017

Licensed: This work is licensed under a Creative Commons Attribution 3.0 License (cc) E E

Publisher:Asian Online Journal Publishing Group
Contribution/Acknowledgement: Both authors contributed to the conception and design of the study.

Funding: This study received no specific financial support.

Competing Interests: The authors declare that they have no conflict of interests.

Transparency: The authors confirm that the manuscript is an honest, accurate, and transparent account of the study was reported; that no vital features of the study have been omitted; and that any discrepancies from the study as planned have been explained.

Ethical: This study follows all ethical practices during writing.

\section{Contents}

1. Introduction

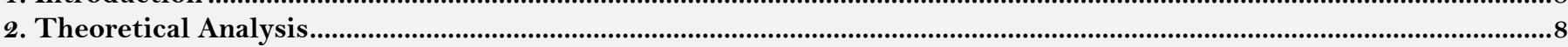

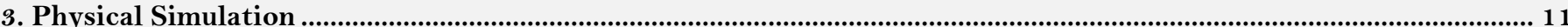

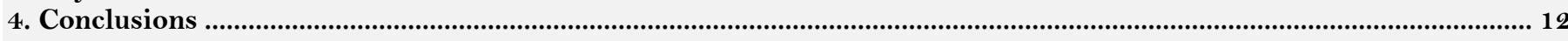

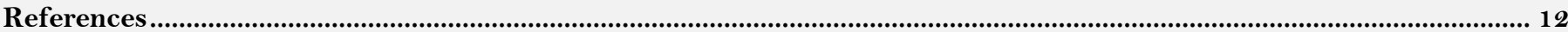




\section{Introduction}

When a laser beam irradiates an object with a statistically inhomogeneous surface, a spatial pattern with a random intensity distribution in the form of individual spots is observed in the reception plane, known as the speckle pattern [1,2]. This picture changes in time when the object moves. Methods for obtaining information on the parameters of motion, based on an analysis of dynamic changes in the spatial structure of the scattered beam, are known as laser speckle-velocimetry (LSV) [3-5]. The technical implementation and physical interpretation of these methods is very diverse. For example, the so-called heterodyne method describes similar dynamic changes in the spatial speckle structure as a result of interference of non-diffracted and diffracted light waves with different frequencies either irregular [6] or regular [7] set of moving scatterers. Because of the difference in frequencies when detecting such a mixture, beats can be distinguished at a difference (Doppler) frequency, which is proportional to the velocity of the object's motion. A very clear and informative description of dynamic changes in speckles on the basis of the Doppler effect is the subject of $[8,9]$.

When a retroreflective sheeting (RRS) is applied to the surface of a moving object as a collection of microscopic glass beads (MGB) [10] the power of the received signal increases, which makes it possible to potentially increase the accuracy of the measurements [11,12]. However, in this case, the spatial characteristics of the scattered light are significantly different from those for rough surfaces or randomly arranged scatterers. Therefore, the specific features of the laser speckle-velocimetry method of objects with RRS require additional analysis.

This work is devoted to the development and investigation of the mathematical and physical models of the LSV method under consideration.

\section{Theoretical Analysis}

Let the surface of the object be a collection of microscopic glass beads (MGB) with an average size $\sigma$ (Fig. 1), and be irradiated from the left to the right by a beam of diameter $\mathrm{a}$.

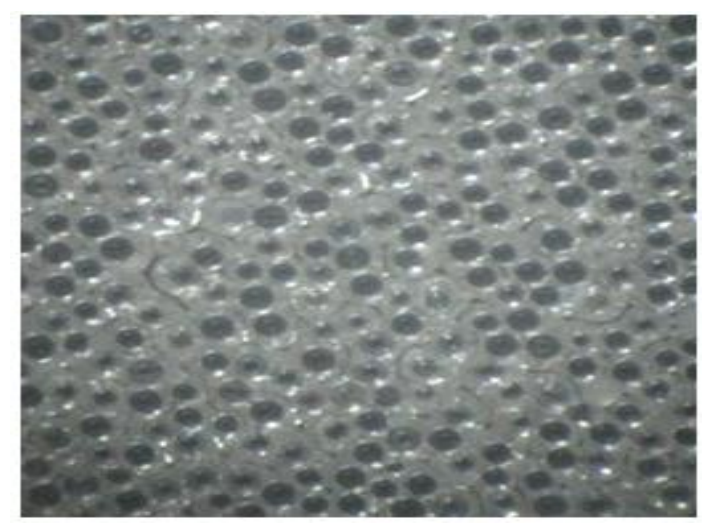

Figure-1. A sheet with microscopic glass beads

The distance between the plane of radiation (observation of the speckle pattern) and the picture plane of the object with the RRS is L, the coordinates of the point in the observation plane are characterized by a radius-vector $\vec{\rho}$, and the coordinates of the point in the picture plane of the object are characterized by the value of the radiusvector $\vec{r}$, the distance between these points is equal to $R(\vec{\rho}, \vec{r})$ (Fig. 2$)$.

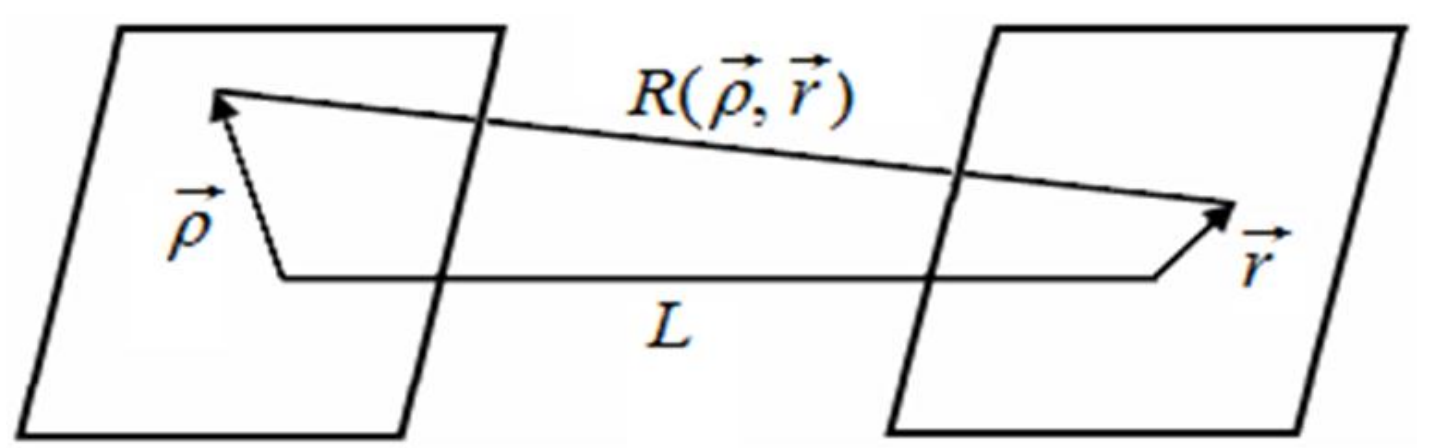

Figure-2. The correspondence between the coordinates of receiving / transmitting and object planes

The incident wave is transformed in the corresponding MGB and then propagates in the opposite direction, forming a speckle pattern in the receiving / transmitting plane. After the transformation on the RRS, the amplitude-phase distribution of the field in the object's image plane can be represented in the form

$$
E(\vec{r})=E_{0} T(\vec{r}) \text {, }
$$

where the factor $T(\vec{r})$ describes the transformation of the incident wave into the reflected one due to propagation of the rays in the RRS elements. 
This transformation takes into account the establishment of a correspondence between the coordinates of the input and output rays, as well as changes in the amplitude and phase of the electromagnetic wave as a result of refractions, reflection and the passage of a certain optical path in a MGB with a given refractive index value. The initial spatial distribution of the amplitude and phase in the incident ray is assumed to be constant.

Then the expression for the field in the observation plane has the form

$$
E(\vec{\rho}, t)=\frac{1}{j \lambda} \int_{S} E_{0} T(\vec{r}) \frac{1}{R(\vec{\rho}, \vec{r})} \exp [-j k R(\vec{\rho}, \vec{r})] d \vec{r},
$$

where $S$ is the area of the illuminated part of the surface.

Now let the object move in the picture plane with velocity $\vec{V}$. Then the complex reflection coefficient will be a function of time $T(\vec{r}-\vec{V} t)$. In the general case this leads to the fact that the distribution of the field in the last expression also becomes a function of time.

Another factor which determines the dependence on the time of the received wave due to Doppler effect. The values of the amplitude and phase of the received wave at any point in the receiving plane are the result of the superposition of the secondary waves emitted by the illuminated RRS region by a beam of diameter a. Since the rays arrive at the receiving point from various points of the object at different angles, then, due to the Doppler effect, the observed frequencies will depend on the magnitude of this angle. The value of the angle correspondently is determined by the set of parameters $\vec{\rho}, \vec{r}, L$.

Taking into account the comments made, it is possible to write down

$$
E(\vec{\rho}, t)=\frac{1}{j \lambda} \int_{S} E_{0} T(\vec{r}-\vec{V} t) \frac{1}{R(\vec{\rho}, \vec{r})} \exp [-j k R(\vec{\rho}, \vec{r})] \exp [2 \pi f(\vec{\rho}, \vec{r}) t] d \vec{r}
$$

Due to the time dependence of the structure of the light field in relation (3), the observed speckle pattern will also vary with time. The intensity at a point with the coordinate $\vec{\rho}$ also depends on time in accordance with the relation

$$
\begin{aligned}
& I(\vec{\rho}, t)=\frac{1}{\lambda^{2}} \int_{S} E_{0} T\left(\overrightarrow{r_{1}}-\vec{V} t\right) \frac{1}{R\left(\vec{\rho}, \overrightarrow{r_{1}}\right)} E_{0}^{*} T^{*}\left(\overrightarrow{r_{2}}-\vec{V} t\right) \frac{1}{R\left(\vec{\rho}, \overrightarrow{r_{2}}\right)} \times \\
& \times \exp \left\{-j k\left[R\left(\vec{\rho}, \vec{r}_{1}\right)-R\left(\vec{\rho}, \overrightarrow{r_{2}}\right)\right]\right\} \exp \left\{j 2 \pi\left[f\left(\vec{\rho}, \overrightarrow{r_{1}}\right)-f\left(\vec{\rho}, \overrightarrow{r_{2}}\right)\right] t\right\} d \overrightarrow{r_{1}} d \overrightarrow{r_{2}}
\end{aligned}
$$

As can be seen from the above relationship, the observed light intensity contains a set of harmonic time oscillations with a Doppler frequency $f_{d}=f\left(\vec{\rho}, \vec{r}_{1}\right)-f\left(\vec{\rho}, \overrightarrow{r_{2}}\right)$, which depends both on the coordinates of the point within the illuminated spot on the object, and on the point in the receiving plane with the coordinate $\vec{\rho}$. The amplitude of these harmonics is also random and is determined by the instantaneous value of the amplitude of the light field in the plane of the object, which is determined by the current value of the function $T(\vec{r}-\vec{V} t)$.

To analyze the value of the Doppler frequency, depending on the observation conditions, we consider the onedimensional case, illustrated in Fig. 3.

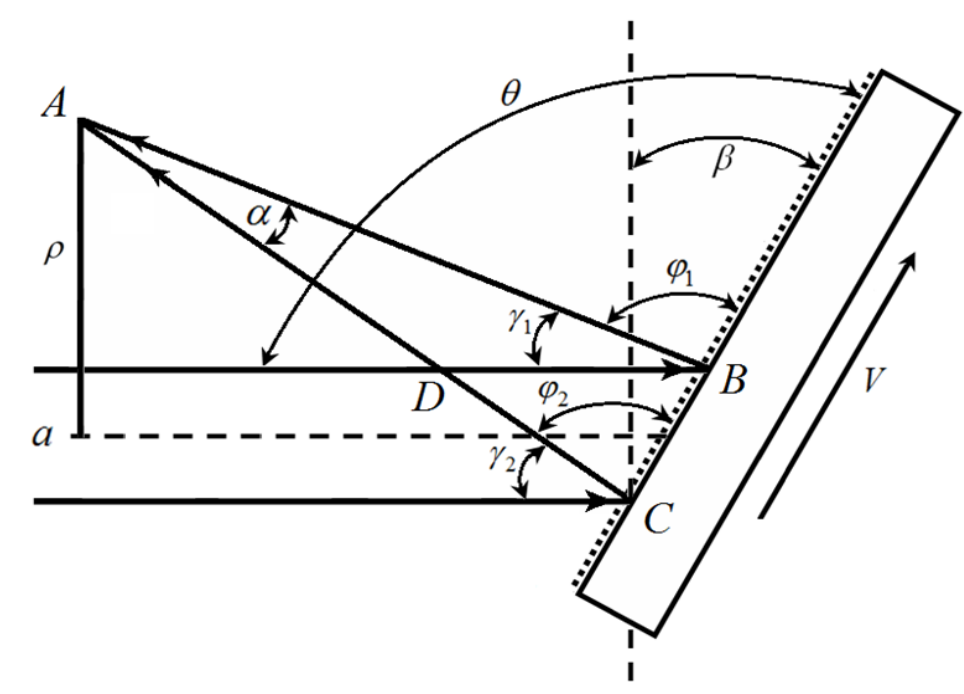

Figure-3. The scheme of beam falling on a moving

Here we have a beam of diameter $a$, incident at an angle $\theta$ to a plane object with an RRS moving with velocity $V$. Here $\beta$ - is the angle between the normal to the ray and the velocity vector, $\varphi_{1}$ and $\varphi_{2}-$ the angles under which the laser radiation is scattered to point $\mathrm{A}$ from various points on the object within the illuminated region.

In accordance with Doppler's law, the difference between the frequencies of waves scattered by the surface and those observed at A does not depend on the angle $\theta$ and is determined only by the values of the angles $\varphi_{1}$ and $\varphi_{2}$

$$
f_{d}=\frac{f V}{c}\left(\cos \varphi_{1}-\cos \varphi_{2}\right)=2 \frac{f V}{c} \sin \frac{\varphi_{1}+\varphi_{2}}{2} \sin \frac{\varphi_{1}-\varphi_{2}}{2} .
$$

We introduce angles $\gamma_{1}$ and $\gamma_{2}$ between the incident and reflected rays. It follows from $\triangle \mathrm{ABD}$ that $\gamma_{2}=\gamma_{1}+\alpha$ Notice, that $\varphi_{1}=\varphi_{2}+\alpha$. 
Then, carrying out obvious trigonometric transformations, we can show that

$$
f_{d}=2 \frac{f V}{c} \sin \frac{\gamma_{1}-\gamma_{2}}{2} \cos \left(\frac{\gamma_{1}+\gamma_{2}}{2}-\beta\right) .
$$

Let us consider the case of a normal incidence of a ray on the surface of an object, when $\beta=0$.

Expression (4) can be represented in the form

$$
I(\rho, t)=\frac{1}{\lambda^{2}} \int_{S} E\left(\gamma_{1}, t\right) E^{*}\left(\gamma_{2}, t\right) \exp \left\{j 2 \pi f_{d}\left(\gamma_{1}, \gamma_{2}\right) t\right\} d \gamma_{1} d \gamma_{2} .
$$

From this relation it is seen that the intensity of light changes in accordance with a continuous set of harmonic oscillations, determined by all possible values $\gamma_{1,2}$. The amplitudes of these harmonics are random and are determined by the instantaneous form of the intensity distribution in the diagram of the scattered radiation on the RRS (Fig. 4a).
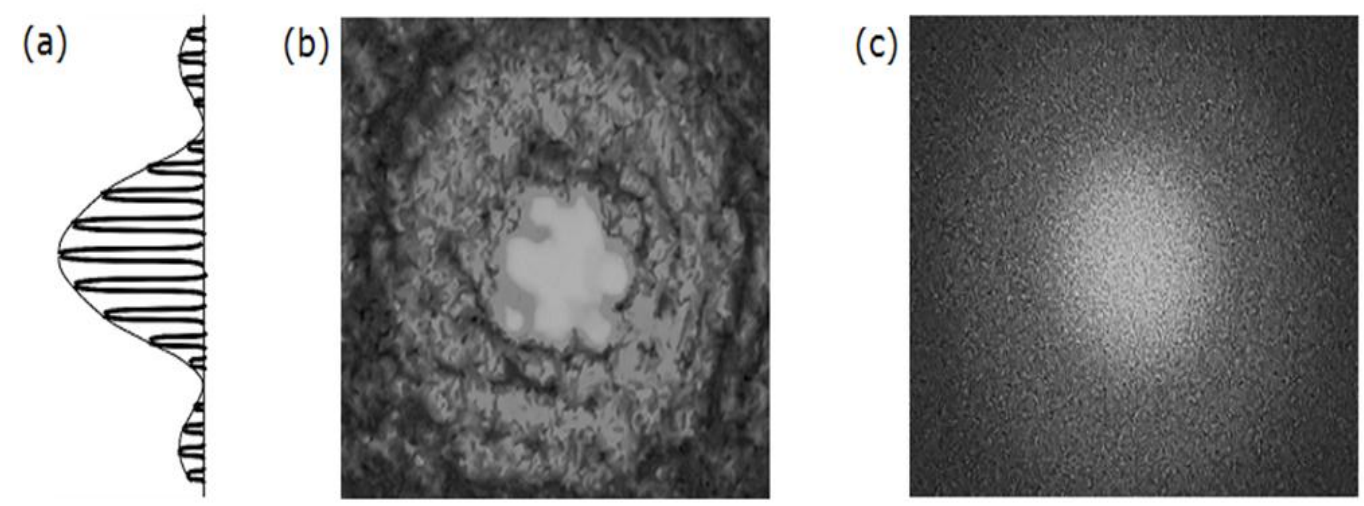

Figure-4. The intensity distribution of the scattered radiation

The shape of the envelope of this distribution is determined by the character of the diffraction of the laser radiation by an average size of a separate microscopic glass beads (Fig. 1), and the internal filling is determined by the current location of the MGB within the area illuminated by the laser beam. This structure is constantly changing in time when the object moves. Figures $4 \mathrm{~b}$ and $4 \mathrm{c}$ show the characteristic instantaneous intensity distributions both in the case of using a relatively small beam diameter (Fig. 4b) and the relatively large diameter of the laser beam (Fig.4c). In the latter case, the number of illuminated MGB is quite large. Obviously, with good averaging over the realizations of the RRS, the resulting intensity distribution will only represent the envelope of the distributions in Fig. 4.

It follows from the patterns of retroreflection that the maximum value of the averaged radiation pattern of the scattered radiation will occur in the direction strictly backward $\left(\gamma_{1,2} \approx 0\right)$. This means that the maximum amplitudes will have oscillations with zero frequency of Doppler shift, because $\sin \frac{\gamma_{1}-\gamma_{2}}{2} \rightarrow 0$.

The frequency band $\delta f_{d}$ is determined by the maximum possible value of the Doppler shift. We note that an appreciable contribution to the observed intensity at point A is caused by oscillations with a noticeable amplitude.

We restrict the region of reception of scattered laser radiation by the angular size within the main lobe of the scattered radiation diagram, i.e. $\gamma_{1,2} \leq \lambda / d \prec \prec 1$.

In the range of these values, we can assume $\cos \frac{\gamma_{1}+\gamma_{2}}{2} \approx 1$.

We also note that $\gamma_{1}-\gamma_{2}=\alpha$ is an angle under which the illuminated region of size a is seen from the point $\mathrm{A}$ at a distance L. In most practical situations it can be assumed $\alpha \approx a / L$.

Therefore $\delta f_{d}=\frac{f V}{c} \frac{a}{L}$. Or otherwise $\delta f_{d}=\frac{V a}{\lambda L}$.

This relation illustrates the fact known from the theory of laser location that when the laser radiation is registered backward, the spectrum of the carrier frequency of the light wave broadens as the velocity and size of the object increase.

Let us now consider the point A at some distance $\rho$ from the axis of the laser beam (Fig. 3). The magnitude of the scattered power in this direction will decrease (Fig. 4) with increasing $\gamma_{1,2}$, but the frequency values themselves will increase in accordance with the relation (5). If we now integrate the received power of the scattered radiation within the receiving aperture and detect it, we obtain a photocurrent that varies with time in accordance with the change in the structure of the reflecting surface $T(\vec{r}-\vec{V} t)$ and all possible variations of the Doppler frequencies. The latter are determined by a search for angles $\gamma$ within the illuminated region and all possible values $\rho$ that vary in the reception plane from zero to the maximum value.

When the ray is inclined to the surface at an angle $\beta$, the relation (5) can be transformed to the form 


$$
f_{d}=2 \frac{f V}{c} \sin \frac{a_{e f f}}{L}\left(\cos \frac{\gamma_{1}+\gamma_{2}}{2} \cos \beta+\sin \frac{\gamma_{1}+\gamma_{2}}{2} \sin \beta\right)=2 \frac{f V a \cos \beta}{c L}\left(1+\sin \frac{\gamma_{1}+\gamma_{2}}{2} \sin \beta\right) .
$$

That is, all Doppler frequencies are shifted in magnitude proportionally $\sin \beta$. Such a shift is naturally absent with a normal incidence of the ray on the surface, this case has been considered above.

\section{Physical Simulation}

The relations obtained above represent a mathematical model of the method of laser speckle-velocimetry of objects with a retroreflective surface. It makes it possible to determine the main regularities of the laser specklevelocimetry process with changing observation parameters. To verify the obtained relationships, the experimental setup shown in Fig. 5 was assembled, which is a physical model of this method.

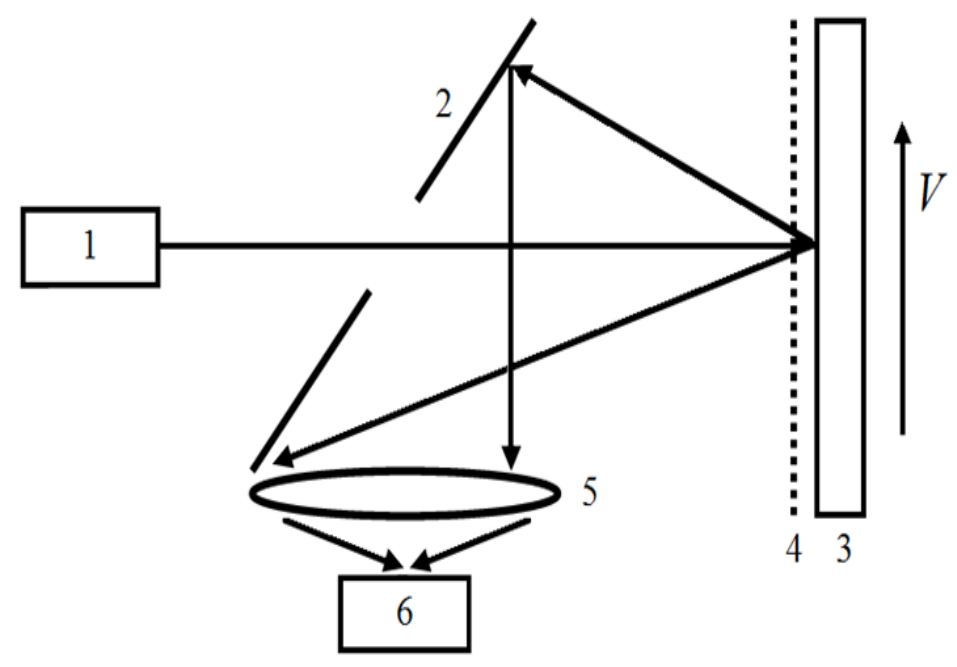

Figure-5. The experimental setup

Here, a laser 1, a mirror 2 with an aperture, a flat object 3 with an RRS 4 deposited on it, a collector lens 5 and a photodetector 6 are depicted. The photocurrent at its output was digitized and its spectrum was further analyzed.

As can be seen from the scheme of the experimental setup, the paraxial beams, which propagate strictly backward within the angles $\gamma_{1,2} \leq \rho / L(\rho \approx a)$, were excluded from reception.

Figure 6a shows the spectrogram of the photocurrent realization on a short time interval, without averaging over the realizations. Under conditions when $\beta \succ 0, V \succ 0$.
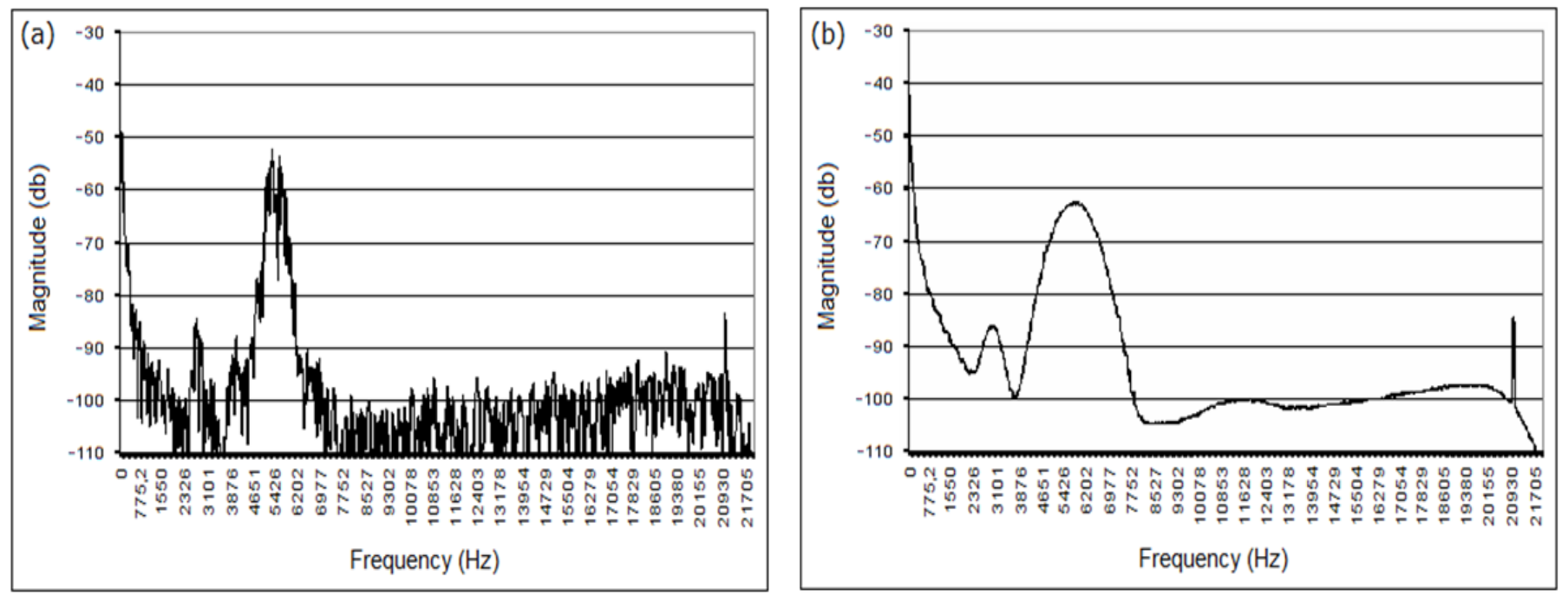

Figure-6. Dependence of photocurrent spectrum on frequency

As can be seen from the figure, its spectrum is randomized due to the presence of recording noise and the random form of the scattering diagram of laser radiation at the RRS (Fig. 4a), see relation (6).

Fig. $6 \mathrm{~b}$ shows the photocurrent spectrum, which is obtained after a good averaging. The curve contains a pronounced localized maximum in the region of $6 \mathrm{kHz}$, which corresponds to the beat of the photocurrent at the Doppler frequency, caused by the movement of the object, i.e. the maximum Doppler frequency. 


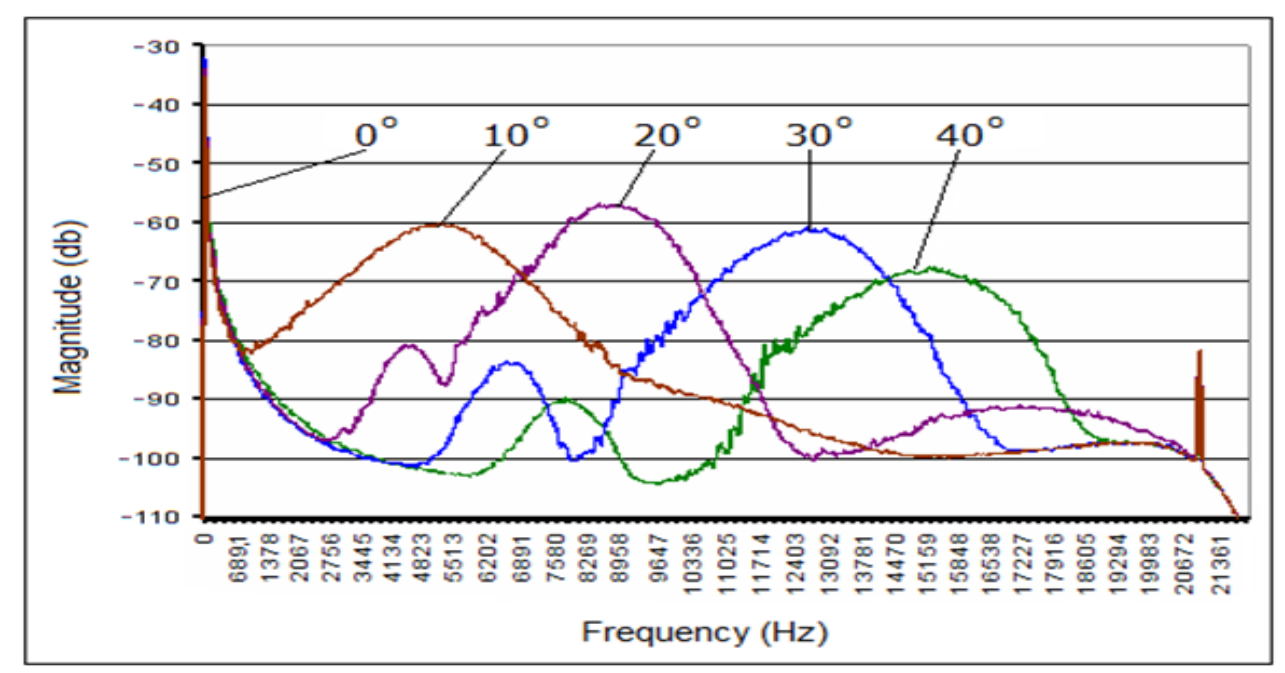

Figure-7. Dependence of photocurrent magnitude on frequency for different incidence angles

Figure 7 shows the regularities of the Doppler frequency shift from zero value at normal incidence of the beam to the surface up to $16 \mathrm{kHz}$ with increasing angle $\beta$. The curves on the diagram are obtained for angles $\beta=0^{\circ}, 10^{\circ}, 20^{\circ}, 30^{\circ}, 40^{\circ}$, respectively. The nature of the change in the Doppler frequency is in good agreement with the mathematical model (7).

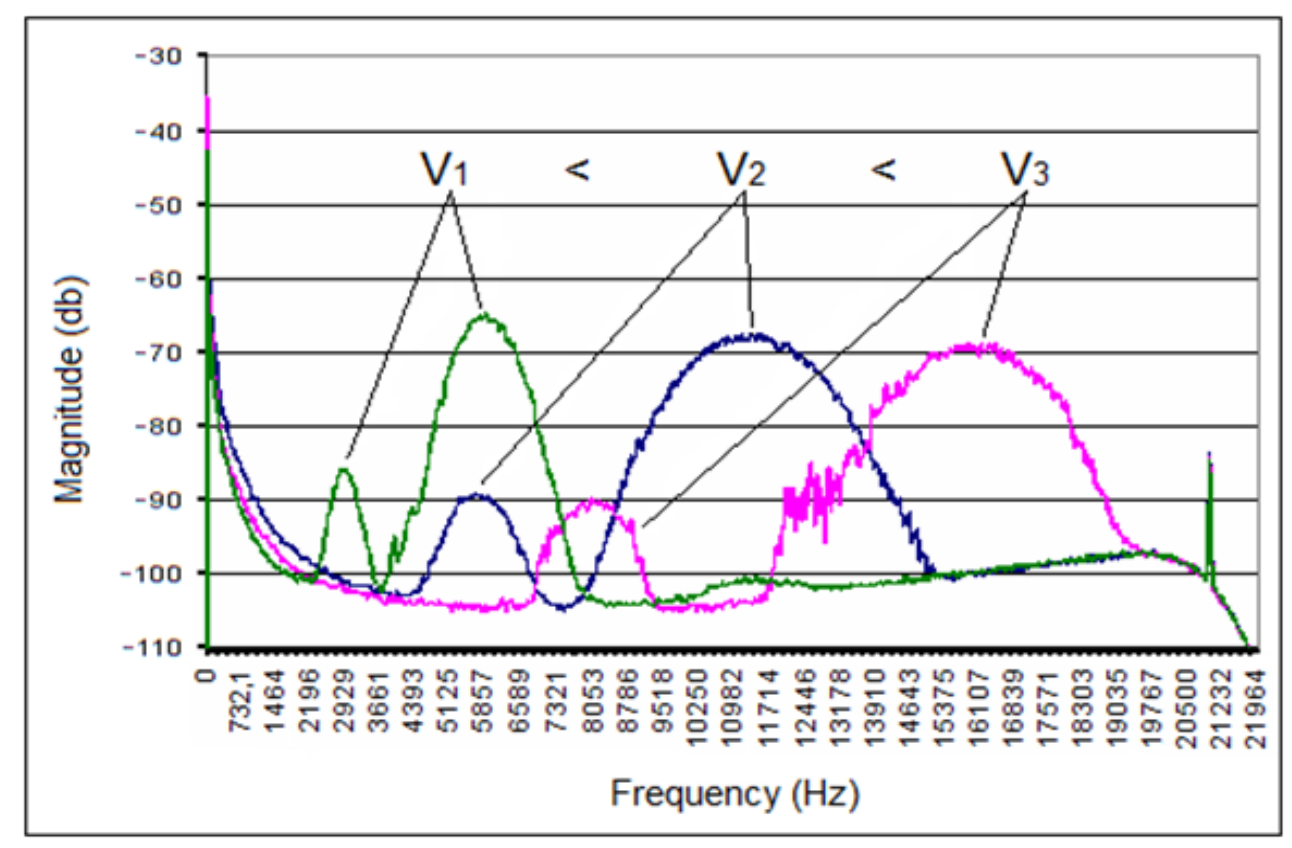

Figure-8. Dependence of photocurrent magnitude on frequency for different values of velocity

Figure 8 shows the patterns of the Doppler frequency change with a change in the speed of motion $\left(\beta=20^{\circ}\right)$. As can be seen from these curves, the Doppler frequency increases with increasing velocity (see relation 7 ). In addition, it can be seen from these curves that as the velocity increases, the spectrum of Doppler frequencies also increases, which was also discussed above. In general, the results of physical modeling are in good agreement with the results of theoretical analysis.

From the above dependences it is clear that this method works quite effectively for angles $\beta$, varying from $10^{\circ}$ to $40^{\circ}$. At smaller angles, the maximum of the Doppler frequency is poorly isolated in the region close to zero frequencies, and at $40^{\circ}$ the power of the received signal is significantly reduced, which is due to the features of retroreflection.

\section{Conclusions}

Thus, the article presents the results of theoretical analysis and physical simulation of the laser Doppler speckle velocimetry method of objects with a retroreflective surface, the results of which are in good agreement. The method consists in irradiating an unfocused beam of a plane object at a small angle, collector receiving of scattered radiation with subsequent detection, and spectral analysis of the recorded photocurrent.

To obtain better results, averaging over object realizations should be performed, which is carried out automatically due to the movement of the object. The maximum value in the spectral distribution corresponds to the Doppler frequency, determined by the velocity of the object's motion. This method is simple in technical implementation and can find application in conditions where it is required to increase the signal-to-noise ratio, for example, when measuring the velocity of remote objects.

\section{References}

[1] M. Francon, Laser speckle and applications in optics: Academic Press. Retrieved: http://b-ok.org/ireader/2217638, 2012.

R. Jones and C. Wykes, Holographic and speckle interferometry: Cambridge University Press. Retrieved: http://b-ok.org, 1989.

H. J. Rabal and J. R. A. Braga, Dynamic laser speckle and applications: CRC Press. Retrieved: https://searchworks.stanford.edu, 2009.

M. Kowalczyk, Laser speckle velocimetry. In: Pluta M, Jabczynski JK, Szyjer M (Eds), Optical velocimetry vol. 2729. Proceedings of the SPIE, 1996. 
[5]

M. Raffel, C. Willert, and J. Kompenhans, Particle image velocimetry. A practical guide: Springer. Retrieved: http://www.springer.com, 1998.

M. D. Alaimo, F. Ferri, and M. A. C. Potenza, "Heterodyne speckle velocimetry," Applied Physics Letters, vol. 88, p. $191101,2006$. View at Google Scholar | View at Publisher

A. Korpel, Acousto-optics. New York: Marcel Dekker, 1988.

S. S. Ulyanov, "Dynamics of speckles with a small number of scattering events: Specific features of manifestation of the doppler effect," Applied Optics, vol. 53, pp. B94-B102, 2014. View at Google Scholar | View at Publisher

S. S. Ulyanov, "Speckle dynamics and Doppler effect," Soros Educational Journal, vol. 7, pp. 109-114, 2001.

J. Lloyd, "A brief history of retroreflective sign face sheet materials. The principles of retroreflection." Reteived: http://www.rema.org.uk/pub/pdf/history-retroreflective-materials.pdf, n.d.

G. N. Dolya and V. Zhyvchuk, "The appreciation of the influence of exactness of focusing on the work of the laser homodyne method of measuring the parameters of vibration," in Proceedings of the SPIE, 2004, pp. 45-52.

G. N. Dolya, A. N. Katunin, O. A. Nad, and A. N. Bulay, "Laser speckle velocimetry for running objects with light reflective surface," Systems of Information Processing, vol. 1, pp. 23-26, 2015. 